\title{
LE GENRE ONCHOCERCA : HYPOTHÊSES SUR SON ÉVOLUTION ET CLÉ DICHOTOMIQUE DES ESPẼCES.
}

\author{
O. BAIN*
}

RÉSUMÉ. Un arbre phylétique et une clé dichotomique des espèces du genre Onchocerca sont proposés.

L'origine du genre et son évolution principale paraissent s'être effectuées en Afrique, où se trouvent l'espèce la plus primitive $(O$. raillieti, chez l'âne domestique) et le plus grand nombre d'espèces (la moitié des 24 représentants du genre).

Cette évolution semble s'être déroulée à une période géologique relativement moderne, correspondant peut-être à l'établissement des Équidés en Afrique, au Pleistocène.

L'espèce humaine, $O$. volvulus, se rattache à une petite lignée d'Onchocerques de Bovidés africains de savane, très évoluée morphologiquement et biologiquement (musculature atrophiée et hypoderme hypertrophié chez la , localisation intranodulaire). $O$. volvulus apparaît comme un parasite encore assez mal adapté à l'homme (microfilaires mal tolérées).

\section{The genus Onchocherca : hypothesis on its evolution and a key to the species.}

SUMMARY. A phylogenetic tree and a dichotomic key to the species in the genus Onchocerca are proposed. The origin and principal evolution have taken place in Africa where the most primitive species (O. raillieti in the donkey) and the greatest number of species (half of the 24 representatives of the genus) occur. This evolution apparently took place in a relatively recent geological period, corresponding perhaps to the establishment of the Equidae in Africa during the Pleistocene.

The species in humans, $O$. volvulus, belongs to a small line of Onchocerca in African Bovidae of the Savanna which is morphologically highly evolved (musculature atrophied and hypodermis hypertrophied in the female, intranodular location). O. volvulus appears to be a parasite which is still not well adapted to man (microfilariae not well tolerated).

La connaissance des Onchocerques a bien progressé depuis une dizaine d'années ; cela tient en bonne partie au fait qu'on dispose d'une technique simple et bon marché (digestion pepsique du matériel fixé à l'alcool $70^{\circ}$ ) pour obtenir des spécimens entiers, même lorsqu'il s'agit de femelles atteignant $1 \mathrm{~m}$ de long. La morphologie du ver peut donc être étudiée aussi soigneusement et complètement que pour n'importe quel Nématode.

* Laboratoire de Zoologie-Vers, associé au CNRS, Museum national d'Histoire naturelle, 61, rue Buffon, F75231 Paris Cedex 05.

Accepté le 20 février I98I. 
Une grande diversité de caractères peut ainsi être utilisée pour distinguer et définir les espèces. Certains d'entre eux ont une valeur phylétique et il devient possible d'envisager d'établir une histoire évolutive des Onchocerques, bien que l'on connaisse certainement peu d'espèces par rapport à celles qui existent réellement, la difflculté de trouver les adultes, quand ils ne sont pas dans des nodules, restant un obstacle important à la connaissance du genre.

\section{Analyse morphologique}

Les caractères utilisés pour apprécier l'évolution du genre Onchocerca sont les suivants :

1. Les papilles cloacales du mâle : il existe initialement 21 papilles disposées suivant le type Spiruride primitif : 1 papille impaire précloacale, 4 paires précloacales, 2 paires postcloacales et 4 paires terminales (cf. Chabaud et Petter, 1961) (fig. 2A). Par la suite, les papilles tendent à se regrouper autour du cloaque et les papilles les plus antérieures et les plus postérieures s'atrophient et disparaissent.

2. La vulve : chez la forme infestante, l'ébauche génitale femelle est peu éloignée de l'anneau nerveux (Bain et Petit, 1978; Bain, 1979); une vulve antérieure chez l'adulte est donc un caractère primitif ; elle tend à migrer en arrière, à hauteur de l'extrémité postérieure de l'œsophage.

3. L'œsophage : il est gros et nettement divisé en portion musculaire et glandulaire chez les formes primitives, plus mince et non divisé chez les formes évoluées ( fig. $1 \mathrm{~A}$; fig. 5B).

4. L'appareil sensoriel céphalique : l'évolution se manifeste par la migration des 4 papilles céphaliques vers l'axe latéral et des 4 papilles labiales externes vers l'axe médian (fig. $1 C$ et $7 C$, in Bain, 1975).

5. Le fourreau épithélio-musculaire de la femelle : les tendances évolutives de ce caractère sont l'atrophie de la musculature, l'hypertrophie de l'hypoderme et principalement des cordes latérales, l'asymétrie liée à la torsion spiralée du ver femelle (Bain et Beveridge, 1979).

6. La cuticule de la femelle : comme chez les autres Nématodes Phasmidiens, la cuticule joue le rôle d'un exosquelette. Dans le cas des femelles d'onchocerques, dont le corps est très long, l'exosquelette tend à être particulièrement résistant. La cuticule est épaissie et divisée en deux couches. La couche externe se renforce par des épaississements transversaux, qui sont de simples ondulations ( $f i g .1 F$ ), ou des côtes bien délimitées (fig. 1E), formant des demi-anneaux interrompus sur les faces latérales; les côtes ont tendance à être de plus en plus sinueuse ( $f i g .4 C$ et D). La couche interne est découpée transversalement par des stries (fig. $1 E$ ), assurant ainsi la souplesse de l'exosquelette.

7. Les microfilaires : elles ont à l'échelle spécifique une valeur très sûre, quoique délicate à manier, en raison de la variabilité des mensurations dues à la diversité des 
techniques utilisées. Par contre, elles n'ont pas de valeur phylétique, pas plus que la nature des vecteurs (Simulies ou Culicoides), parfois différents pour des espèces proches.

8. Les spicules : ils n'ont également qu'une valeur spécifique. Le spicule droit se termine par une partie renflée, la spatule, dont la forme varie ( $f i g .3 \mathrm{~A} a ̀ \mathrm{D}$ ), et qui fait sur la face dorsale une saillie plus ou moins marquée, le talon. Les dimensions des spicules ont souvent une valeur spécifique peu sùre, car elles peuvent varier fortement pour une espèce donnée (par exemple, O. reticulata Diesing, 1841).

\section{Classification des espèces}

a) Parmi les 24 espèces suffisamment connues, une seule présente des caractères morphologiques franchement primitifs; il s'agit d'O. raillieti Bain et coll., 1976, parasite de l'âne domestique en différentes régions d'Afrique.

b) L'espèce du dromadaire, O. fasciata Railliet et Henry, 1910, présente de l'Afrique au Pakistan, reste relativement proche de raillieti, mais sa cuticule est plus complexe (Bain et Nasher, 1981).

c) Un groupe de 3 espèces, $O$. reticulata Diesing, 1841, $O$. cervicalis Railliet et Henry, 1910, et $O$. bohmi (Supperer, 1953), parasites du cheval domestique, particulièrement en Europe, conserve également une morphologie assez primitive et, en particulier, une vulve bien antérieure.

d) L'espèce parasite de Suidé en Malaisie, $O$. dewittei Bain et coll., 1977, est également de type assez primitif, mais avec un œsophage déjà nettement atrophié (fig. 5B):

e) Enfin, le grouge O. sonini Bain et coll., 1978, O. lerouxi Bain et coll., 1978, et O. beaveri Bain et coll., 1978, parasite des Bovidae de forêt d'Afrique Centrale, constitue le dernier groupe conservant des éléments morphologiques proches de raillieti, tels que vulve antérieure et œsophage bien divisé (fig $5 \mathrm{~A}$ ).

f) En Afrique également, mais, cette fois, réparties dans les savanes et encerclant le bloc forestier congolais, existent chez les Bovidae toute une série d'espèces homogènes, qui vivent dans des nodules et ont une morphologie nettement plus évoluée (œsophage à différenciation glandulaire à peine marquée, hypoderme hypertrophié, musculature atrophiée) (fig. 13A).

Deux espèces se trouvent chez les Bovins domestiques: $O$. ochengi Bwangamoi, 1969, dans le derme, et $O$. dukei Bain et coll., 1974, dans le conjonctif sous-cutané et périmusculaire,

Deux autrès espèces viennent d'être découvertes chez des Bovidae sauvages, O. schulzkeyi Denké et coll., 1981, chez Hippotragus equinus, et $O$. hamoni Denkéet coll., 
1981, chez Kobus (Adenota) kob; toutes deux sont un peu plus spécialisées que les précédentes.

L'espèce humaine, $O$. volvulus (Leuckart, 1893), également évoluée, ressemble par là aux espèces de Bovidae sauvages, mais elle semble devoir être rattachée en fait à $O$. ochengi, en raison de la similitude des microfilaires.

g) En Asie et Australie se trouvent deux autres espèces nodulaires : $O$. cebei Galliard, 1937 (= O. sweetae Spratt et Moorhouse, 1976) ${ }^{1}$, dans le derme du Bubale, qui semble être vicariant d'O. ochengi, et $O$. gibsoni Cleland et Johnston, 1910, dans le conjonctif sous-cutané et périmusculaire de Bos taurus et B. indicus, qui semble être vicariant d'O. dukei.

h) Les Bovins domestiques, en Afrique et en Asie, hébergent $O$. armillata Railliet et Henry, 1909, espèce atypique, qui présente un mélange de caractères primitifs et évolués.

i) Ils hébergent également, en Europe principalement, mais aussi dans diverses régions du monde, un groupe formé par $O$. lienalis Stiles, 1892, et $O$. gutturosa Neumann, 1910, primitifs par la vulve antérieure, mais relativement évolués pour les autres caractères (œsophage mince, atrophie des papilles cloacales assez marquée, migration des papilles céphaliques et labiales).

j) Le peuplement en Onchocerques des Cervidés européens paraît être également polyphylétique, avec, d'une part, $O$. flexuosa (Wedl, 1856), espèce très originale, mais conservant cependant des éléments primitifs (grand œsophage divisé, vulve antérieure), et le groupe O. jakutensis (Gubanov, 1964) (=O. tubingensis Bain et SchulzKey, 1974) ${ }^{1}$, O. tarsicola Bain et Schulz-Key, 1974, et O. garmsi Bain et Schulz-Key, 1976, bien spécialisé (par exemple, vulve très éloignée de l'anneau nerveux) ( $f i g .9 B$, $C, D)$.

k) La dernière espèce, $O$. cervipedis Wehr et Dikmans, 1935, parasite de Cervidés néarctiques, a un œsophage grêle et une vulve le plus souvent postœsophagienne (Wehr et Dikmans, 1977) et paraît dériver du groupe précédent.

\section{Conclusions phylétiques}

L'arbre phylétique proposé (fig. 18), en s'appuyant sur ces données, est donc d'un type assez particulier, car il semble déterminé par la répartition géographique des parasites plutôt que par la place zoologique des hôtes. Ainsi, les Suidés et les

I. La mise en synonymie de sweetae et de cebei est restée longtemps en suspens car le matériel type de cebei semble perdu, et sa description originale fait mention de papilles nettement précloacales qui n'existent pas chez sweetae.

Les données récentes (BArN et coll., I980) montrant que des spécimens identifiables à sweetae existent en Inde et à Bangkok chez le Buffle domestique, font que l'existence d'une espèce distincte chez le même animal au Tonkin est très peu vraisemblable. Nous acceptons donc la synonymie.

2. L'unique caractère différentiel précédemment utilisé pour séparer jakutensis et tubingensis était la taille du spicule gauche (BArN et Schulz-KEY, I974). 
Camélidés, dont les représentants modernes sont apparus bien avant ceux des Équidés, n'hébergent pas les onchocerques les plus primitives.

La naissance du genre et son évolution principale paraissent s'être effectuées en Afrique, où se trouvent l'espèce la plus primitive et le plus grand nombre d'espèces ; elle semble s'être déroulée à une période géologique relativement très moderne, correspondant peut-être à l'établissement des Équidés en Afrique, au Pléistocène.

\section{Hypothèses épidémiologiques tirées de la phylogénie}

Les conclusions épidémiologiques que l'on pourrait tirer de cet arbre phylétique sont les suivantes :

1 - Le genre Onchocerca est un genre moderne, en cours de spéciation, et on doit s'attendre à découvrir encore de très nombreuses espèces.

2 - L'espèce humaine, $O$. volvulus, est extrêmement proche de certaines espèces connues chez les Bovidae africains de savanes, et, en particulier, d'O. ochengi. Il faut la considérer comme un parasite encore relativement mal adapté à l'homme, ce qui explique vraisemblablement pourquoi les microfilaires sont aussi mal tolérées.

3 - Cependant, il ne semble pas que le nodule soit assimilable à une réaction de défense de l'organisme vis-à-vis d'un parasite mal toléré. O. volvulus et les autres formes nodulaires de Bovidés constituent une petite lignée homogène, dont le fourreau épithélio-musculaire est très spécialisé, et les espèces morphologiquement plus primitives dont elles dérivent ne se développent pas dans des nodules.

Le nodule apparaît donc comme une formation induite par ces espèces d'onchocerques, et favorable à leur développement, car, comme l'indiquaient Railliet et Henry, 1910, les onchocerques sont fondamentalement des parasites des tissus conjonctifs.

4 - L'existence de petits foyers d'onchocercose humaine dans des régions très isolées telles que l'Amazonie pouvait inciter à rechercher l'existence de réservoirs animaux dans ces régions.

La phylogénie des onchocerques ne paraît pas favorable à cette hypothèse.

En effet, d'une façon générale, dans l'évolution d'une lignée, le pouvoir adaptatif et la faculté de spéciation du parasite par capture d'un nouvel hôte s'observe le plus souvent près de son lieu d'origine (cf., par exemple, le genre Heligmosomum, Trichostrongylide d'Arvicolidae, étudié par Durette-Desset, 1967).

Dans le cas des onchocerques, il serait étonnant qu'une spéciation d'un parasite chez l'homme ait lieu en dehors de l'Afrique, centre d'évolution et de dispersion du genre. L'hypothèse la plus logique est donc un isolement récent de l'onchocerque humaine en Amérique ; mais ces 3 siècles d'isolement sont largement suffisants pour permettre la réalisation de phénomènes de subspéciation (cf., par exemple, les cas de subspéciations observés avec le genre Heligmosomoides par Durette-Desset et coll., 1972). 


\section{CLÉ DES ESPÈCES.}

1 - (10) Esophage $>2 \mathrm{~mm}$ de long ${ }^{1}$ et portion glandulaire nettement différenciée, plus épaisse que la portion musculaire. (fig. $1 \mathrm{~A}$ ).

2 - (9) Distance vulve-tête inférieure ou égale au double de la distance anneau nerveux-tête (fig. 1A).

Microfilaire dépourvue de cou étroit en arrière de la tête ( $f$ ig. $1 B$ et $C$ ).
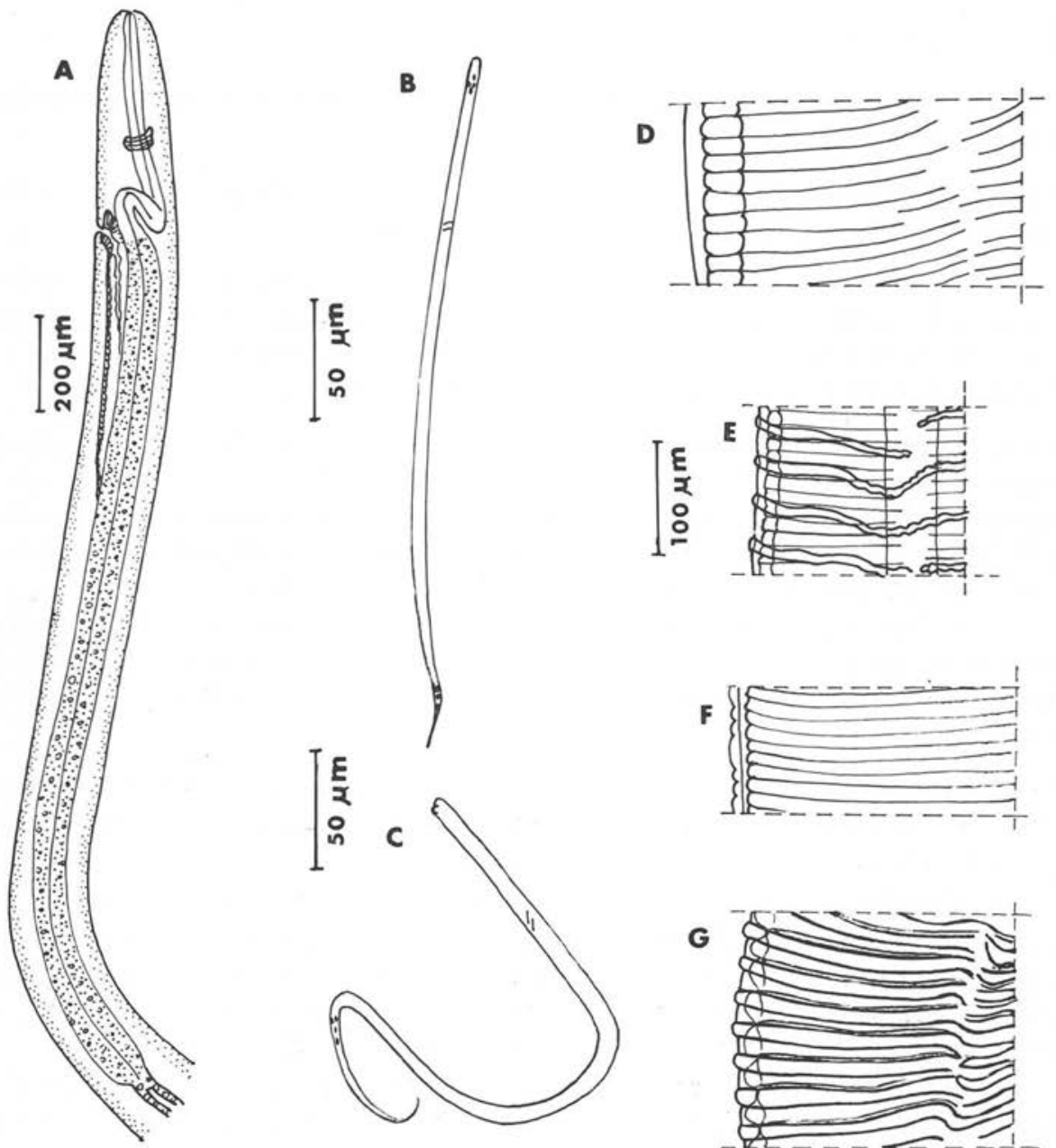

FIG. I. - A : O. cervicalis, , région antérieure, vue latérale; $\mathrm{B}:$ idem, microfilaire ; $\mathrm{C}: 0$. reticulata, microfilaire; D à $\mathrm{G}$, cuticule $ᄋ$, vue latérale (éch. : Ioo um); $\mathrm{D}: O$. vaillieti $; \mathrm{E}$ : $O$. cervicalis; $\mathrm{F}: O$. flexuosa; $\mathrm{G}: O$. reticulata. de long.

I. Chez O. cervicalis, quelques rares spécimens ont un œsophage légèrement inférieur à $2 \mathrm{~mm}$ 
3 - (4) Couche externe de la cuticule 우 sans côtes ni ondulations transversales (fig. 1D).

Dix paires de papilles caudales, disposées suivant le type spiruride (4 paires précloacales, 2 paires postcloacales, groupe terminal de 4 paires plus les phasmides près de la $9^{\mathrm{e}}$ paire) ( $f i g .2 \mathrm{~A}$ ).

Cuticule de la 우 épaisse avec rides longitudinales externes et stries internes très nettes.

Spicules longs (gauche 440-500 $\mu \mathrm{m}$; droit : 150-170 $\mu \mathrm{m})$.

Microfilaire longue de $180-215 \mu \mathrm{m}$ sur $4 \mu \mathrm{m}$ de large.

Parasite sous-cutané de l'âne domestique Equus asimus, en Afrique.

O. raillieti Bain, Muller, Khamis, Guilhon, Schillhorn van Veen, 1976.

4 - (3) Couche externe de la cuticule + avec côtes ( $f i g .1 E$ et $G$ ) ou avec ondulations transversales ( fig. $1 F$ ).

Moins de dix paires de papilles caudales, celles-ci non disposées suivant le type Spiruride (fig. $2 B, C, D$ ).

5 - (8) Cuticule $q$ avec côtes.

Paires de papilles caudales préclocales présentes.

6 - (7) Côtes aussi larges que les intercôtes et non sinueuses (fig. 1G).

Une strie par intercôte.

Six paires de papilles volumineuses régulièrement alignées de part et d'autre de la région cloacale; groupe terminal réduit à une petite paire de papilles (fig. 2B).

Microfilaire à long filament caudal (fig. 1C).
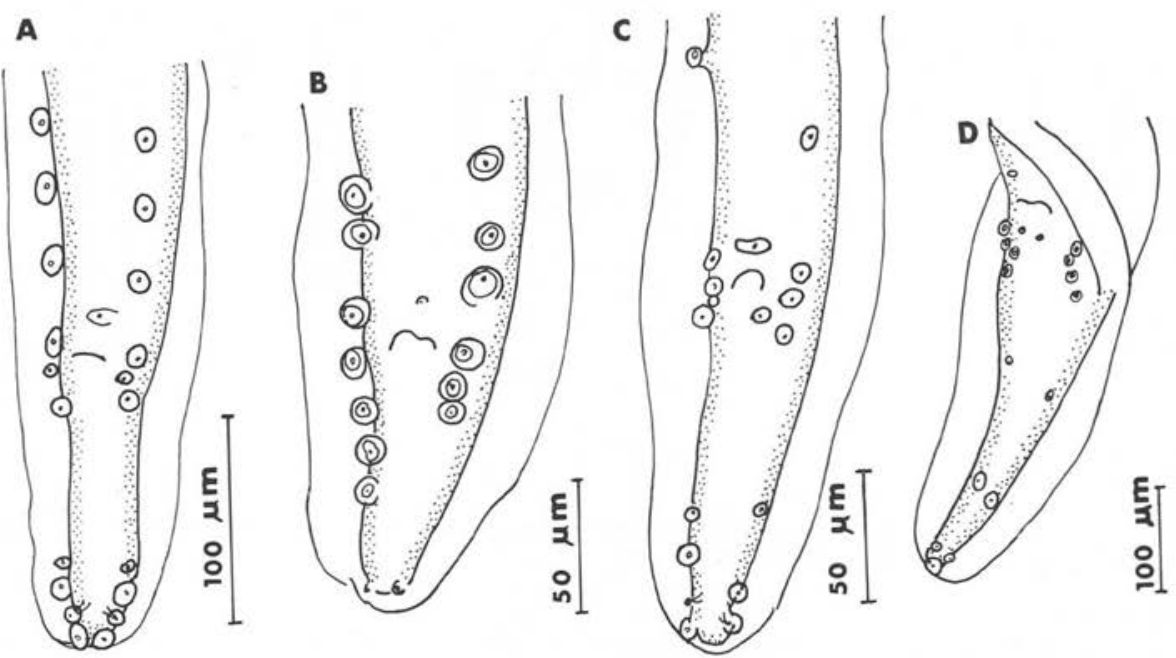

FIG. 2. - Queue $\hat{O}$, vue ventrale; $\mathrm{A}: O$. raillieti $; \mathrm{B}: O$. veticulata $; \mathrm{C}: O$. cervicalis $; \mathrm{D}: O$. flexuosa 
Enroulement du corps de la ㅇ en ressort.

Spicule gauche long de 200 à $340 \mu \mathrm{m}^{1}$.

Spicule droit long de 120-145 $\mu \mathrm{m}$, à talon peu marqué (fig. $3 \mathrm{~A}$ ).

Microfilaire longue de 310-395 $\mu \mathrm{m}$ sur 6-7 $\mu \mathrm{m}$ de large ${ }^{2}$.

Parasite du ligament suspenseur du boulet chez le cheval domestique,

Equus caballus, en Europe, Amérique du Nord, Asie, Australie...

O. reticulata Diesing, 1841,

7 - (6) Côtes plus étroites que les intercôtes et sinueuses sur les faces latérales (fig. 1E).

Trois stries par intercôte.

Cinq paires de papilles non volumineuses dans la région cloacale disposées irrégulièrement (un groupe de 4 paires et une paire nettement plus antérieure) ; groupe terminal de 3 paires de papilles ( $f i g .2 C$ ).

Microfilaire à queue courte (fig. $1 B$ ).
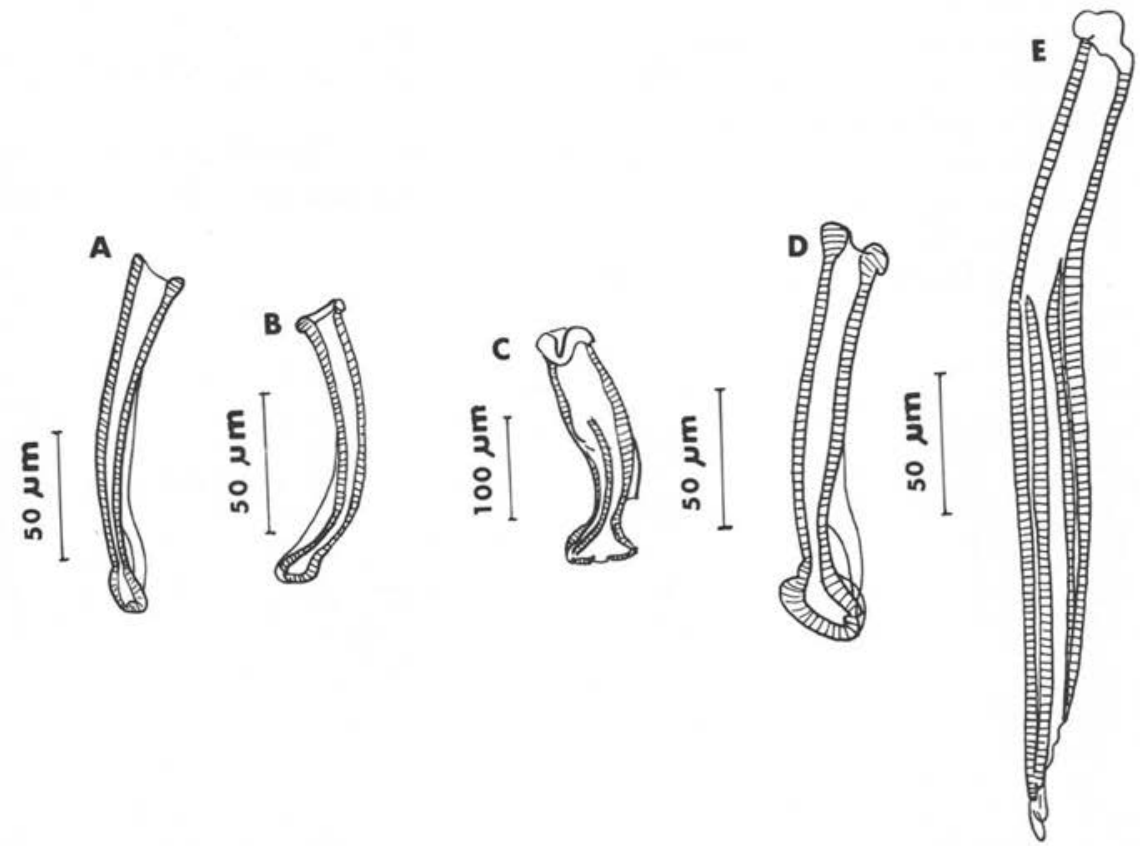

FIG. 3. - A à D, spicule droit ; A : O. reticulata ; B : O. cervicalis ; $: O$. flexuosa ; D : O. armillata ; $\mathrm{E}:$ spicule gauche d'O. armillata.

I. Pour les espèces anciennement connues, les dimensions données pour les spicules sont celles que nous avons observées; elles n'ont qu'une valeur indicative et ne donnent pas les longueurs extrèmes qui ont pu être observées par les divers auteurs.

2. Les dimensions des microfilaires sont prises à frais (immobilisation à la chaleur) ou, quand cela n'est pas possible, sur les microfilaires utérines mûres situées près de la vulve des 우 fixées. 
Spicule gauche long de 340 à $380 \mu \mathrm{m}$.

Spicule droit long de 100-125 $\mu \mathrm{m}$, à talon formant un angle droit (fig. 3B).

Microfilaire longue de $210-250 \mu \mathrm{m}$ sur 4-5 $\mu \mathrm{m}$ de large.

Parasite du ligament cervical du cheval domestique Equus caballus, en Europe, Amérique du Nord, Amérique du Sud, Asie, Australie...

O. cervicalis Railliet et Henry, 1910.

8 - (5) Cuticule q sans côtes mais avec alternance régulière d'une grande et de deux petites ondulations transversales ( fig. $1 F$ ).

Pas de paires de papilles caudales en avant du cloaque (fig. 2D).

Dimensions très grandes de l'œsophage (ㅇ : 7-10 mm; to : 4-7 mm), de la queue (ㅇ: : 700-1500 $\left.\mu \mathrm{m} ; \sigma^{\uparrow}: 400-500 \mu \mathrm{m}\right)$, des spicules (gauche : $600-900 \mu \mathrm{m}$; droit : $200-270 \mu \mathrm{m})$.

Spicule droit à crochet très puissant ( fig. $3 \mathrm{C}$ ).

Microfilaire longue de 220-245 $\mu \mathrm{m}$ sur 4-4, $4 \mu \mathrm{m}$ de large.
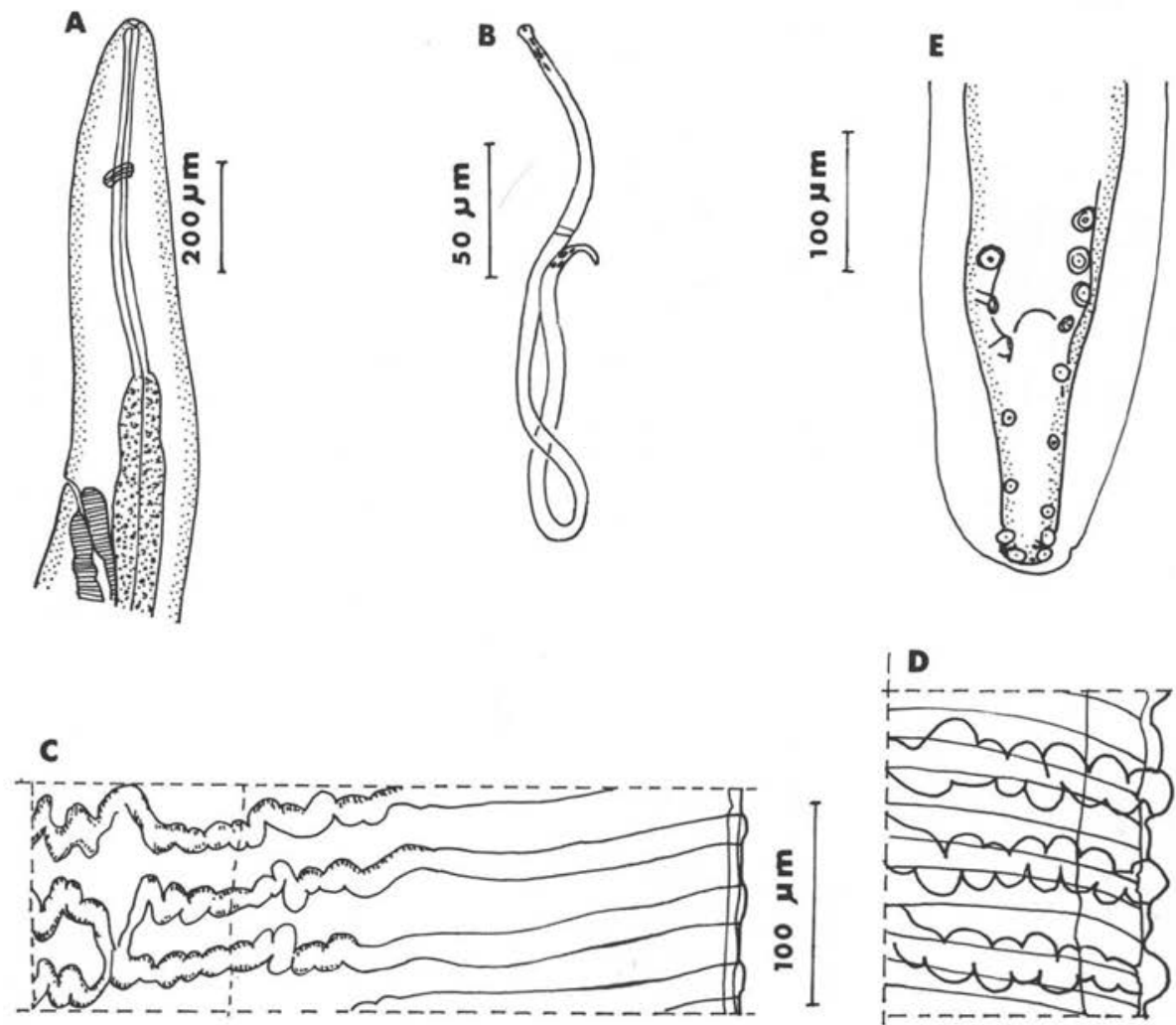

FIG. 4. - O. armillata; $\mathrm{A}$ : région antérieure 9 , vue latérale; $\mathrm{B}:$ microfilaire ; $\mathrm{C}$ et $\mathrm{D}$ : cuticule , vue latérale, respectivement au milieu du corps et à $4 \mathrm{~cm}$ de la tête; $\mathrm{E}:$ queue $\hat{\sigma}$. 
Parasite sous-cutané dorsal intranodulaire du Cerf, Cervus elaphus, en Europe.

O. flexuosa (Wedl, 1856).

9 - (2) Distance vulve-tête égale au triple de la distance anneau nerveux-tête (fig. 4A).

Microfilaire avec cou étroit en arrière de la tête ( $f i g .4 B$ ).

Corps de la 우 très large $(500 \mu \mathrm{m})$ aux circonvolutions situées dans un seul plan.

Côtes très ondulées sur les faces latérales.

Deux stries par intercôte et une strie par côte (fig. 4D).
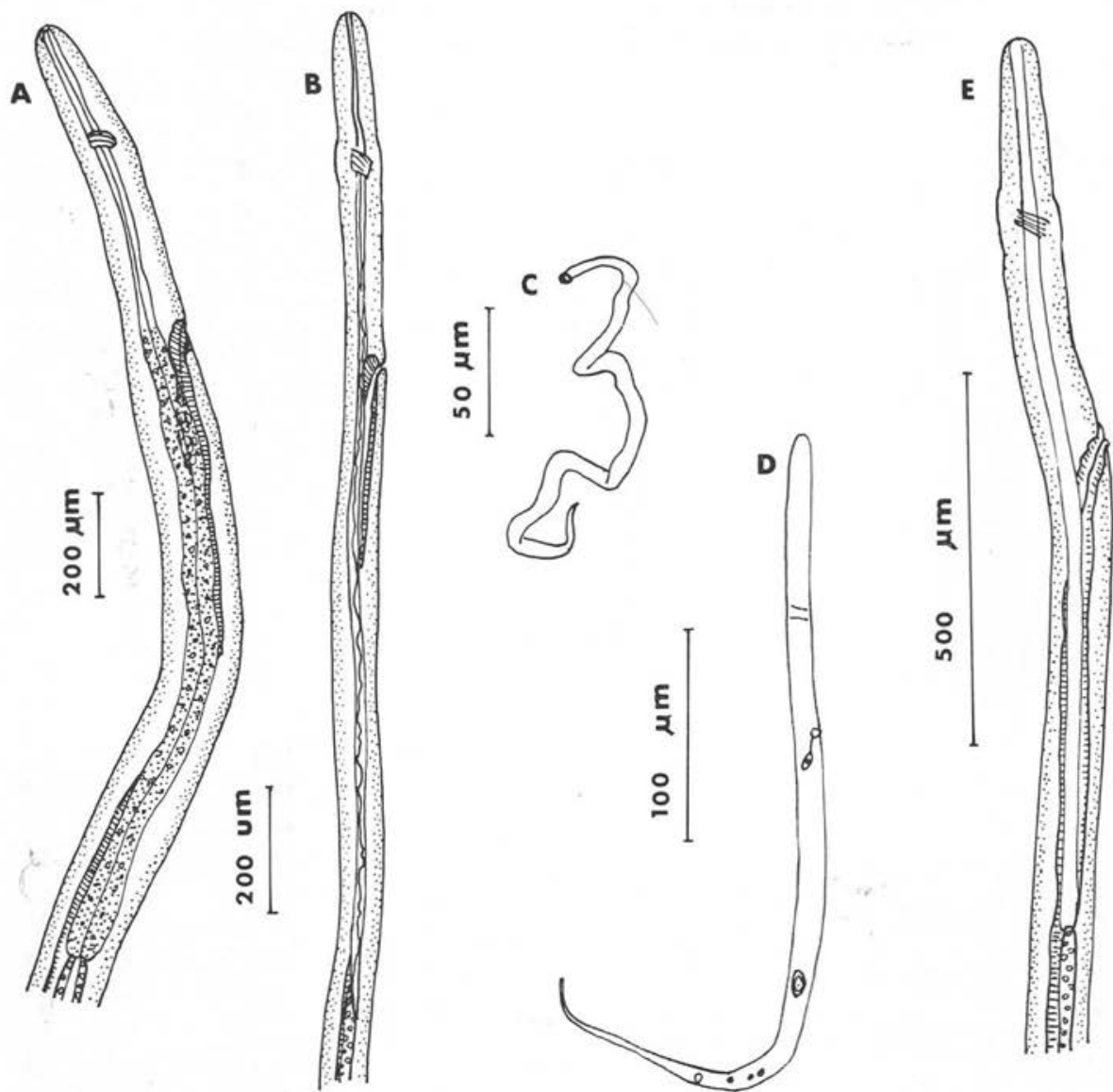

FrG. 5. - A : O. lerouxi 오, région antérieure; $\mathrm{B}: O$. dewittei, 우, idem; $\mathrm{C}:$ O. sonini, microfilaire; D : O. bohmi, microfilaire; $\mathrm{E}: 0$. bohmi, , région antérieure (D et E, in Supperer, 1953). 
Paires de papilles caudales alignées presque régulièrement, sans séparation entre le groupe cloacal et le groupe terminal ( $f i g .4 E$ ).

Spicule gauche long de $300 \mu \mathrm{m}$ en moyenne à manche court et à lame épaisse ( $\mathrm{fig}$. 3E).

Spicule droit long de $140 \mu \mathrm{m}$ en moyenne, au talon formant un angle droit (fig. 3D).

Microfilaire longue de 305-360 $\mu \mathrm{m}$ sur 5-6 $\mu \mathrm{m}$ de large.

Parasite de la paroi de l'aorte des Bovins, Bos taurus, B. indicus et Bubalus bubalis, en Afrique, en Inde et en Asie du Sud-Est, plus rarement des Caprins et Ovins domestiques en Inde.

O. armillata Railliet et Henry, 1909.

10 - (1) Esophage $<2 \mathrm{~mm}$, divisé ( fig. $5 \mathrm{~A}$ ) ou non divisé ( $\mathrm{fig} .5 \mathrm{~B}$ ).

11 - (18) Esophage $>1 \mathrm{~mm}$ et à portion glandulaire nettement différenciée dans les deux sexes.

Distance vulve-tête double ou triple de la distance anneau nerveux-tête. Groupe terminal de papilles caudales de 3-4 paires (fig. 6).

12 - (13) Côtes très saillantes, très ondulées et coudées ou bifurquées sur les faces latérales (fig. 7A).

Corps de la + épais $(400-475 \mu \mathrm{m})$.

ô de grande taille $(9 \mathrm{~cm})$.

A

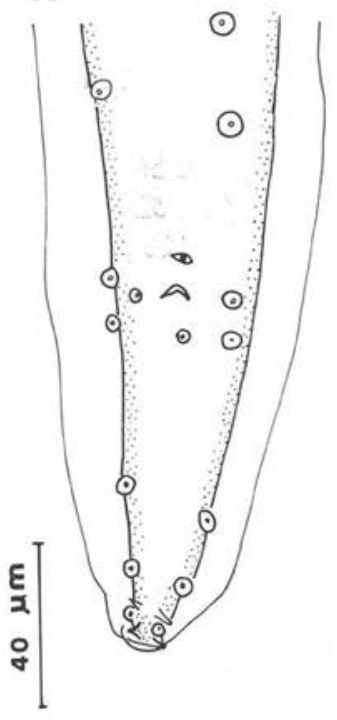

B

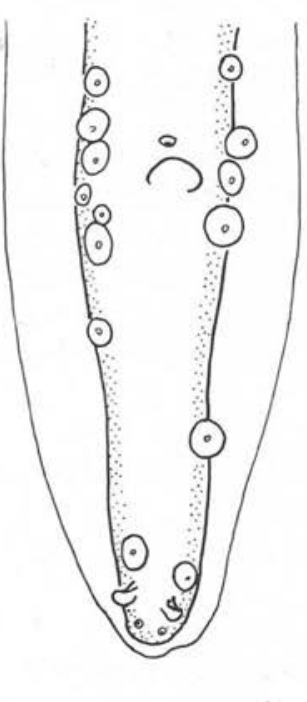

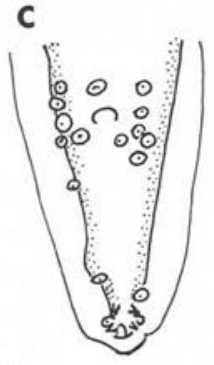

D

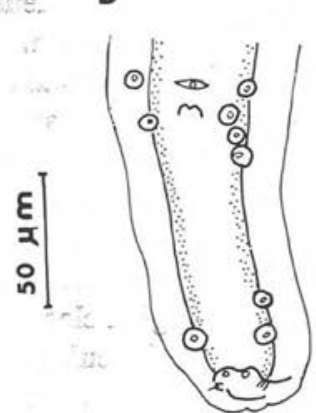

Fig. 6. - Queue $\hat{\jmath}$, vue ventrale; A : O. sonini ; B : O. fasciata ; C : O. beaveri; D : O. lerouxi. 

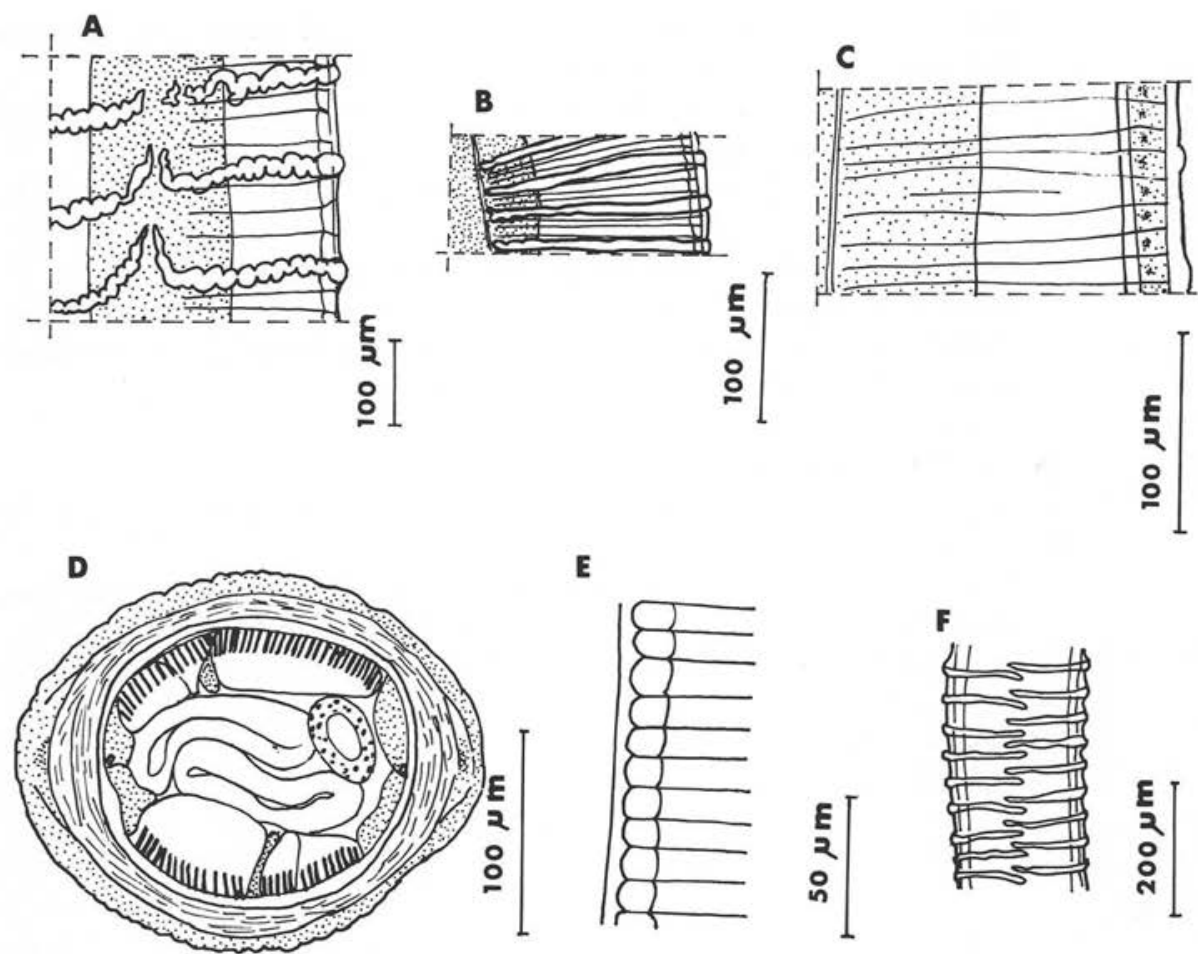

E
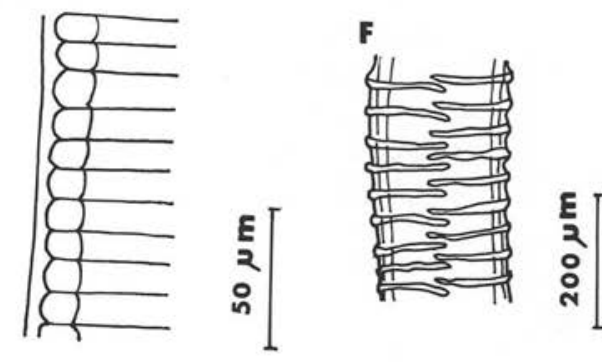

FIg. 7. - A à C, cuticule , vue latérale; A : O. fasciata ; B : O. beaveri ; C : O. sonini ; D :

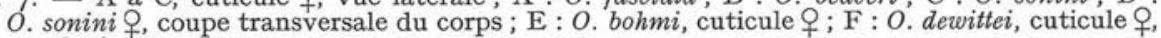
vue latérale.

Huit ou neuf paires de papilles caudales, dont la $10^{\mathrm{e}}$ paire bien visible ; pas de paire de papilles médioventrales post-cloacales (fig. $6 \mathrm{~B}$ ).

Spicules gauche et droit longs en moyenne de $350 \mu \mathrm{m}$ et $120 \mu \mathrm{m}$.

Microfilaire longue de 235-260 $\mu \mathrm{m}$ sur 3, 5-3,6 $\mu \mathrm{m}$ de large.

Parasite sous-cutané de la tête et du cou, plus rarement à la base de la queue, chez le dromadaire, Camelus dromedarius, de l'Afrique au Pakistan.

O. fasciata Railliet et Henry, 1910.

13 - (12) Côtes peu saillantes, droites ou à peine ondulées et ni coudées ni bifurquées sur les faces latérales ( fig. $7 B$ ).

Corps de la o moins épais $(250-325 \mu \mathrm{m})$.

$\hat{o}$ petit $(<3 \mathrm{~cm})$.

14 - (15) Trois stries par intercôte (fig. 7B).

Région cloacale avec 5-6 paires de papilles groupées (fig. 6C).

Spicule gauche long de $250 \mu \mathrm{m}$.

Spicule droit long de $85 \mu \mathrm{m}$, au talon très peu marqué.

Microfilaire longue de 248-262 $\mu \mathrm{m}$ sur 3,5 $\mu \mathrm{m}$ de large. 
Parasite des ligaments de l'articulation axis-atlas chez un Cephalophe,

Cephalophus monticola, en forêt équatoriale africaine (Gabon).

O. beaveri Bain, Chabaud, Landau, 1978.

15 - (14) Quatre stries par intercôte (fig. 7C).

Région cloacale avec moins de 5 paires de papilles groupées (fig. 6D) ou avec papilles espacées ( fig. 6A).

16 - (17) Une ou deux paires de papilles nettement antérieures au cloaque (fig. 6A). Microfilaire à capuchon céphalique cuticulaire ( $f i g .5 C$ ) et à région antérieure très amincie.

Pas de torsion spiralée du corps de la + autour de l'axe longitudinal.

Cuticule $q$ épaisse et musculature épaisse ( fig. 7D).

Spicule gauche long de $180-213 \mu \mathrm{m}$.

Spicule droit long de $88-98 \mu \mathrm{m}$ à talon peu marqué.

Microfilaire longue de $235 \mu \mathrm{m}$ sur $7 \mu \mathrm{m}$ de large.

Parasite du conjonctif sous-cutané des métacarpes et métatarses chez un

Céphalophe, Cephalophus nigrifrons, en forêt équatoriale africaine (Gabon).

O. sonini Bain, Chabaud, Landau, 1978.

17 - (16) Pas de papilles nettement antérieures au cloaque (fig. 6D).

Microfilaire sans capuchon céphalique cuticulaire et à région antérieure à peine amincie.

Torsion spiralée du corps de la $q$ autour de l'axe longitudinal.

Cuticule de la $q 2$ à 3 fois moins épaisse.

Spicule gauche long de $270 \mu \mathrm{m}$.

Spicule droit long de $90 \mu \mathrm{m}$, au talon formant un angle droit.

Mircofilaire longue de 255-290 $\mu \mathrm{m}$ sur 3-3,5 $\mu \mathrm{m}$ de large.

Parasite des aponévroses des muscles du thorax, de l'abdomen et des cuisses d'un Céphalophe, $C$. nigrifrons, en forêt équotariale africaine (Gabon).

O. lerouxi Bain, Chabaud, Landau, 1978.

18 - (11) Esophage n'ayant pas à la fois les deux caractères précédents.

19 - (20) Microfilaire à long filament caudal (fig. 5D).

Couche externe de la cuticule $ᄋ$ sans côtes ni ondulations transversales (fig. 7E).

Renflement cervical chez la 우.

Distance vulve-tête égale au double de la distance anneau nerveuxtête (fig. 5E).

Spicule gauche long de 275-300 $\mu \mathrm{m}$.

Spicule droit long de $95-100 \mu \mathrm{m}$, à talon saillant ${ }^{1}$.

Microfilaire longue de 300-330 $\mu \mathrm{m}$ sur 6-7 $\mu \mathrm{m}$ de large ${ }^{2}$.

I. D’après Supperer, 1953.

2. Ibid. 
Parasite de la paroi des artères et des veines des métacarpes et métatarses du cheval domestique, E. caballus, en Europe.

O. bohmi (Supperer, 1953).

20 - (19) Microfilaire à filament caudal court.

21 - (22) Cuticule de la 우 avec côtes se chevauchant sur les faces latérales ( fig. $7 F$ ).

Côtes rectilignes; couche interne de la cuticule sans stries.

Région antérieure de la $q$ très mince.

Renflement cervical présent ( $f i g .5 B$ ).

Spicule gauche long de 215-250 $\mu \mathrm{m}$.

Spicule droit long de $80-85 \mu \mathrm{m}$, au talon formant un angle droit.

Microfilaire longue de 228-247 $\mu \mathrm{m}$ et large de $6,5 \mu \mathrm{m}$.

Parasite dans le conjonctif sous-cutané et les tendons des métacarpes et métatarses du sanglier, Sus scrofa jubatus, en Asie du Sud-Est (Malaisie).

O. dewittei Bain, Ramachandran, Petter et Mak, 1977.

22 - (21) Cuticule de la $ᄋ$ avec côtes ne se chevauchant pas sur les faces latérales.

23 - (24) Intercôtes très étroites, donnant l'aspect d'une cuticule striée ( fig. 8A).

Région antérieure de la $q$ épaisse ( fig. 9A).

Renflement cervical plus ou moins net.

Vulve le plus souvent à la fin, rarement à mi-hauteur, de l'œsophage.

Spicule gauche long de 225-245 $\mu \mathrm{m}$.

Spicule droit long de 105-112 $\mu \mathrm{m}$.

Microfilaire longue de $224 \mu \mathrm{m}$ sur $6 \mu \mathrm{m}$ de large ${ }^{1}$.

Parasite sous-cutané des métacarpes et métatarses et des oreilles de Cervidés, Odocoileus sp. p., en Amérique du Nord.

O. cervipedis Wehr et Dikmans, 1935.

A

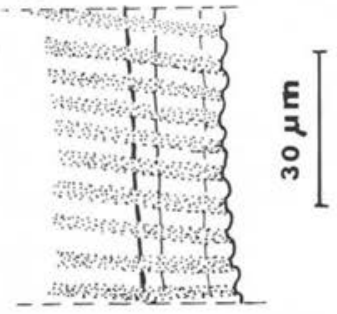

B
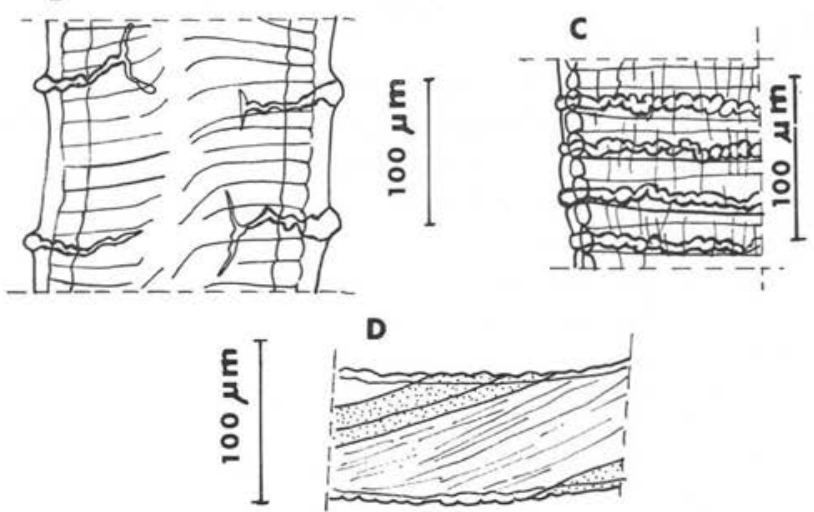

D

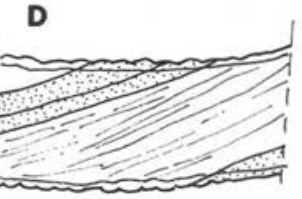

FIG. 8. - Cuticule , vue latérale; A : O. cervipedis; B : O. gutturosa ; $: O$. lienalis, région postérieure; $\mathrm{D}: \mathrm{O}^{\prime}$. lienalis, torsion du corps.

I. D'après Hibler, 1965 . 

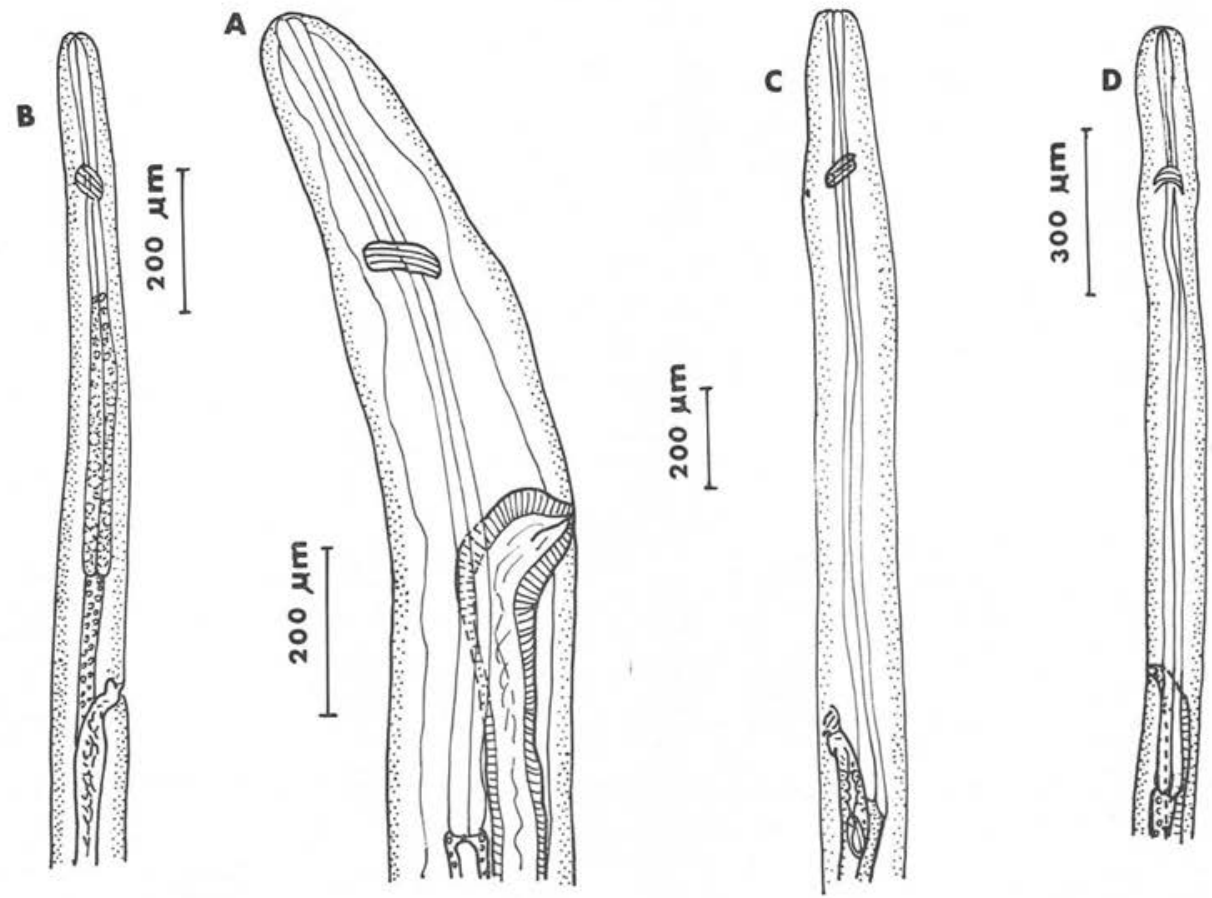

FIG. 9. - Région antérieure 우, vue latérale; A : O. cervipedis ; B : O jakutensis ; C : O. garmsi ; D : O. tarsicola.

24 - (23) Intercôtes larges.

25 - (34) Musculature + développée et cordes latérales étroites.

26 - (29) Distance vulve-tête égale à deux-trois fois la distance anneau nerveux-tête.

27 - (28) Côtes très saillantes, souvent bifurquées près du plan latéral, sinueuses sur les faces latérales.

Quatre stries par intercôtes, parfois plus ( $f i g .8 B$ ).

Couche externe de la cuticule + sans rides longitudinales (fig. 10A).

Ailes latérodorsales chez la ㅇ.

Pas de torsion spiralée du corps de la o autour de l'axe longitudinal.

Région caudale de la o en général très enroulée ( fig. 11A).

Queue du ô avec un groupe cloacal de 3-4 paires et un groupe terminal de 3 paires, la $9^{\mathrm{e}}$ transformée en pointe cuticulaire ( $f i g .12 \mathrm{~A}$ ).

Spicule gauche long de 270-290 $\mu \mathrm{m}$.

Spicule droit long de $85 \mu \mathrm{m}$.

Microfilaire longue de 225-270 $\mu \mathrm{m}$ sur 3,7-4 $\mu \mathrm{m}$ de large ( $f$ g. $10 \mathrm{~B}$ ).

Parasite du ligament cervical, de la scapula, du trochanter et du grasset chez les Bovins, Bos taurus et Bos indicus dans le monde entier. O. gutturosa Neumann, 1910. 
A
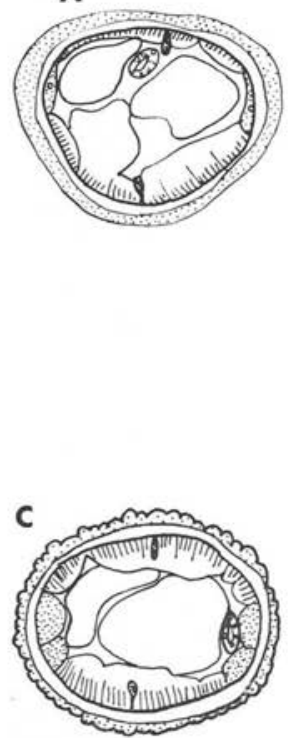
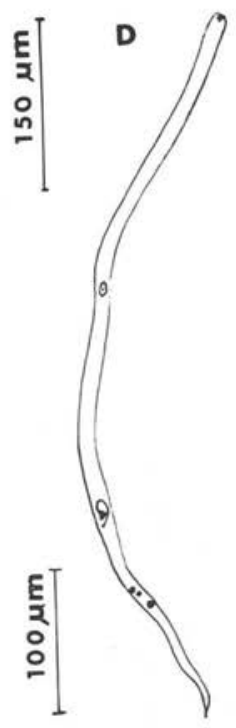

B

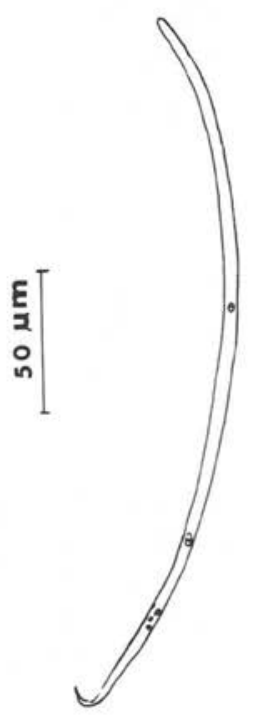

FIG. Io. - A et B : O. gutturosa, coupe transversale du corps $\$$ et microfilaire; $\mathrm{C}$ et $\mathrm{D}: O$. lienalis, idem et idem.
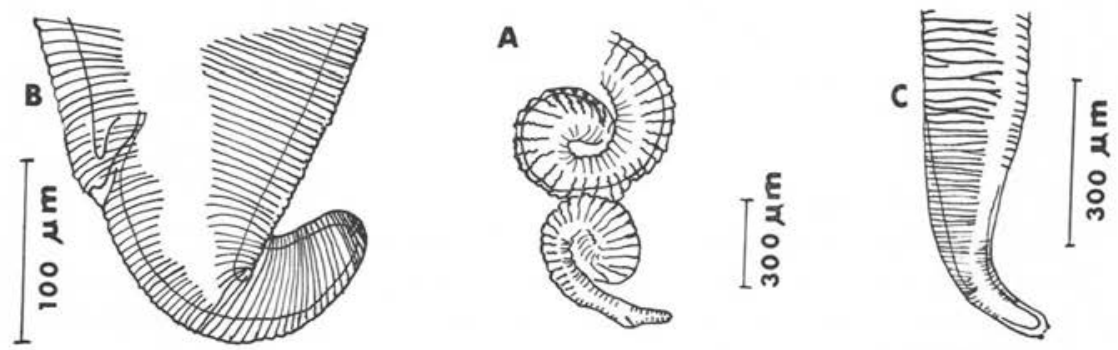

FIG. II. - Région caudale , vue latérale; A : O. gutturosa; B : O. garmsi ; C : O. tarsicola.

28 - (27) Côtes faiblement marquées sauf dans la région postérieure; à ce niveau côtes très sinueuses sur les faces médianes et latérales (fig. $8 \mathrm{C}$ ). et non bifurquées.

Deux stries par intercôtes.

Couche externe de la cuticule o avec rides longitudinales (fig. 10C).

Pas d'ailes chez la 우.

Torsion spiralée du corps de la 우 autour de l'axe longitudinal (fig. 8D).

Queue du $\hat{o}$ avec un groupe cloacal de 3-4 paires de papilles et un groupe terminal de 4 paires normales.

Spicule gauche long de $220 \mu \mathrm{m}$.

Spicule droit long de $75 \mu \mathrm{m}$.

Microfilaire longue de 225-270 $\mu \mathrm{m}$ et large de 4,9-5,3 $\mu \mathrm{m}$ (fig. 10D). 
Parasite du ligament grastrosplénique des Bovins, Bos taurus et Bos indicus, en Europe, Amérique, Asie, Australie ; inconnu en Afrique.

O. lienalis Stiles, 1892.

29 - (26) Distance vulve-tête supérieure à 4 fois la distance anneau nerveux-tête (fig. 9B, C, D).

30 - (31) Esophage divisé et très court $(600 \mu \mathrm{m}-750 \mu \mathrm{m})$ (fig. 9B).

Côtes saillantes, un peu ondulées, souvent bifurquées sur les faces latérales.

Trois stries par intercôte.

Neuvième paire de papilles caudales transformée en petits tubercules; dixième paire absente (fig. 12B).

Spicule gauche long de $270-310 \mu \mathrm{m}$.

Spicule droit long de $100-110 \mu \mathrm{m}$.

Microfilaire longue de $248-295 \mu \mathrm{m}$ et large de 5,8-6,5 $\mu \mathrm{m}$ à tête trapézoïdale ou arrondie-oblique suivant l'orientation.
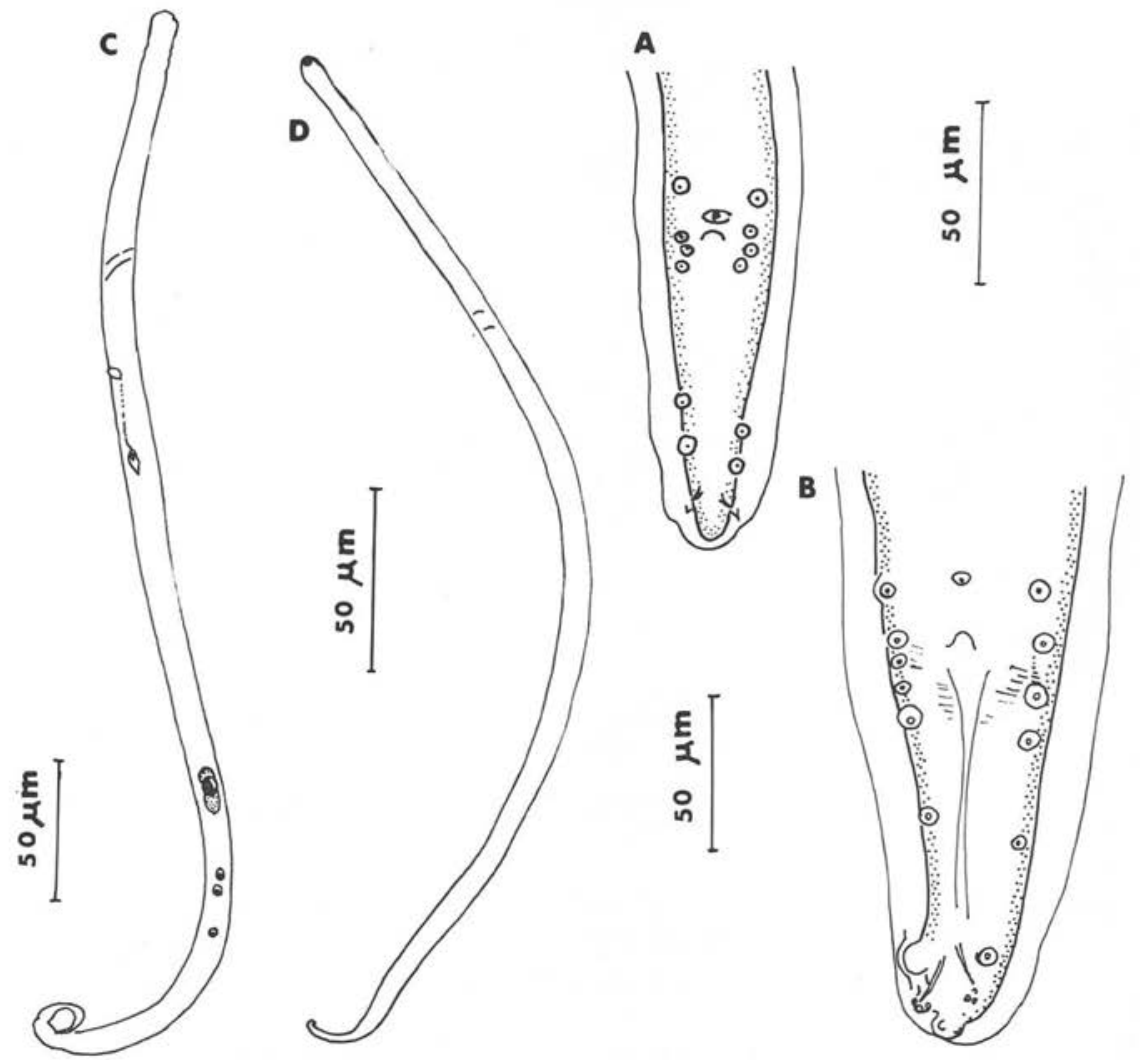

FIG. I2. - A et B, queue; $\mathrm{A}: O$. gutturosa ; $\mathrm{B}: O$. jakutensis ; $\mathrm{C}$ et D, microfilaires ; $:$ O. tarsicola $; \mathrm{D}:$ O. garmsi. 
Parasite sous-cutané dorsal intranodulaire du cerf Cervus elaphus, en Europe.

O. jakutensis (Gubanov, 1964) (= O. tubingensis Bain et Schulz-Key, 1794).

31 - (30) Esophage non divisé long de plus d'1 mm.

32 - (33) Microfilaire très épaisse (10-12 $\mu \mathrm{m})$ et dépourvue de cou (fig. 12C).

우 à région antérieure mince $(100 \mu \mathrm{m})$ (fig. 9D) et à queue cylindrique (fig. 11C).

Côtes saillantes, non bifurquées sur les faces latérales.

Quatre stries par intercôtes.

Spicule gauche long de 200-250 $\mu \mathrm{m}$.

Spicule droit long de 95-130 $\mu \mathrm{m}$.

Microfilaire longue de $400-430 \mu \mathrm{m}$.

Parasite sous-cutané au niveau des articulations radio-carpale et tibiotarsale du Cerf, Cervus elaphus, du Renne, Rangifer tarandus, en Europe. O. tarsicola Bain et Schulz-Key, 1974.
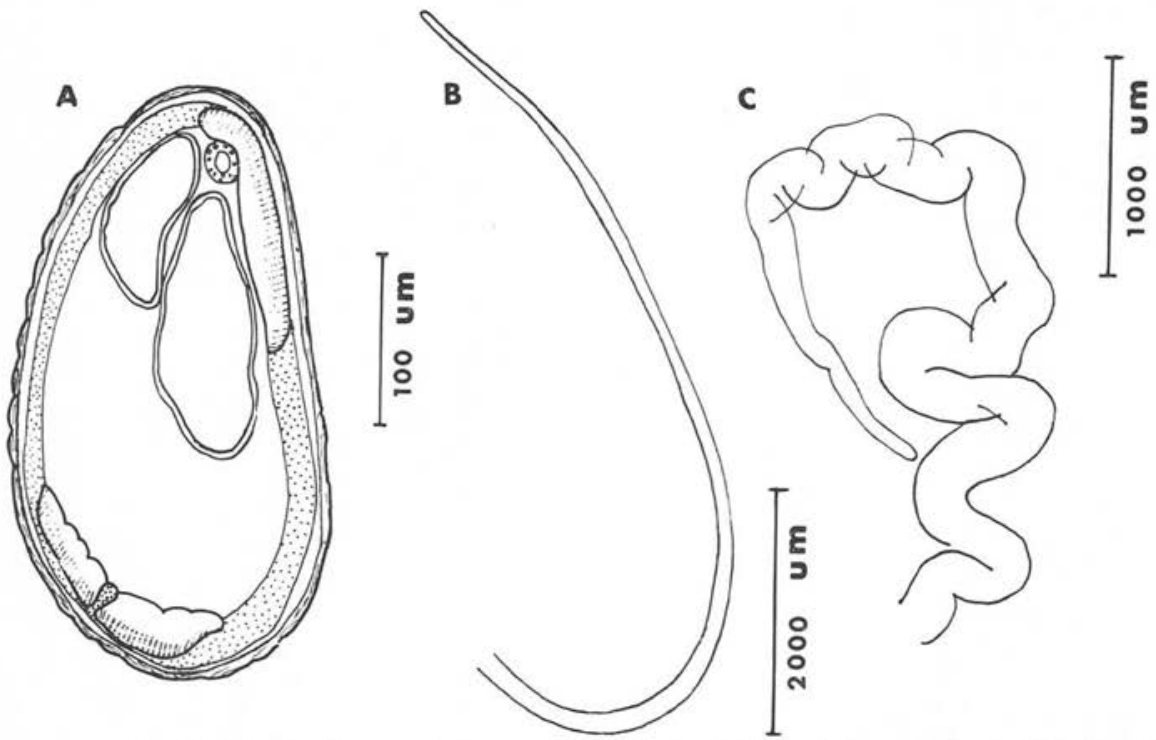

Fig. I3. - A : O. volvulus, 우, coupe transversale du corps ; B et C, région antérieure 9 de $O$. schulzkeyi et O. hamoni.

33 - (32) Microfilaire moins épaisse (7,5-8,5 $\mu \mathrm{m}$ ) pourvue d'un cou (fig. 12D).

ㅇa région antérieure épaisse $(180 \mu \mathrm{m})$ ( $f$ ig. $9 \mathrm{C}$ ) et à queue conique ( $\mathrm{fig} .11 \mathrm{~B}$ ) 우 à région antérieure épaisse $(180 \mu \mathrm{m})$ ( fig. $9 C$ ) et à queue conique (fig. 11B).

Côtes saillantes, non bifurquées sur les faces latérales.

Trois stries par intercôtes. 
$\delta$ inconnu.

Microfilaire longue de 287-330 $\mu \mathrm{m}$.

Parasite du conjonctif sous-cutané ventral du Cerf, Cervus elaphus, en Europe.

O. garmsi Bain et Schulz-Key, 1976.

34 - (25) Musculature + atrophiée et cordes latérales très larges (égales ou supérieures aux champs musculaires) (fig. 13A).

Vers enroulés à l'intérieur de nodules.

35 - (40) Cuticule ㅇ lisse ou ondulée, suivant les portions du corps observées mais pas de côtes (fig. 14A, B).
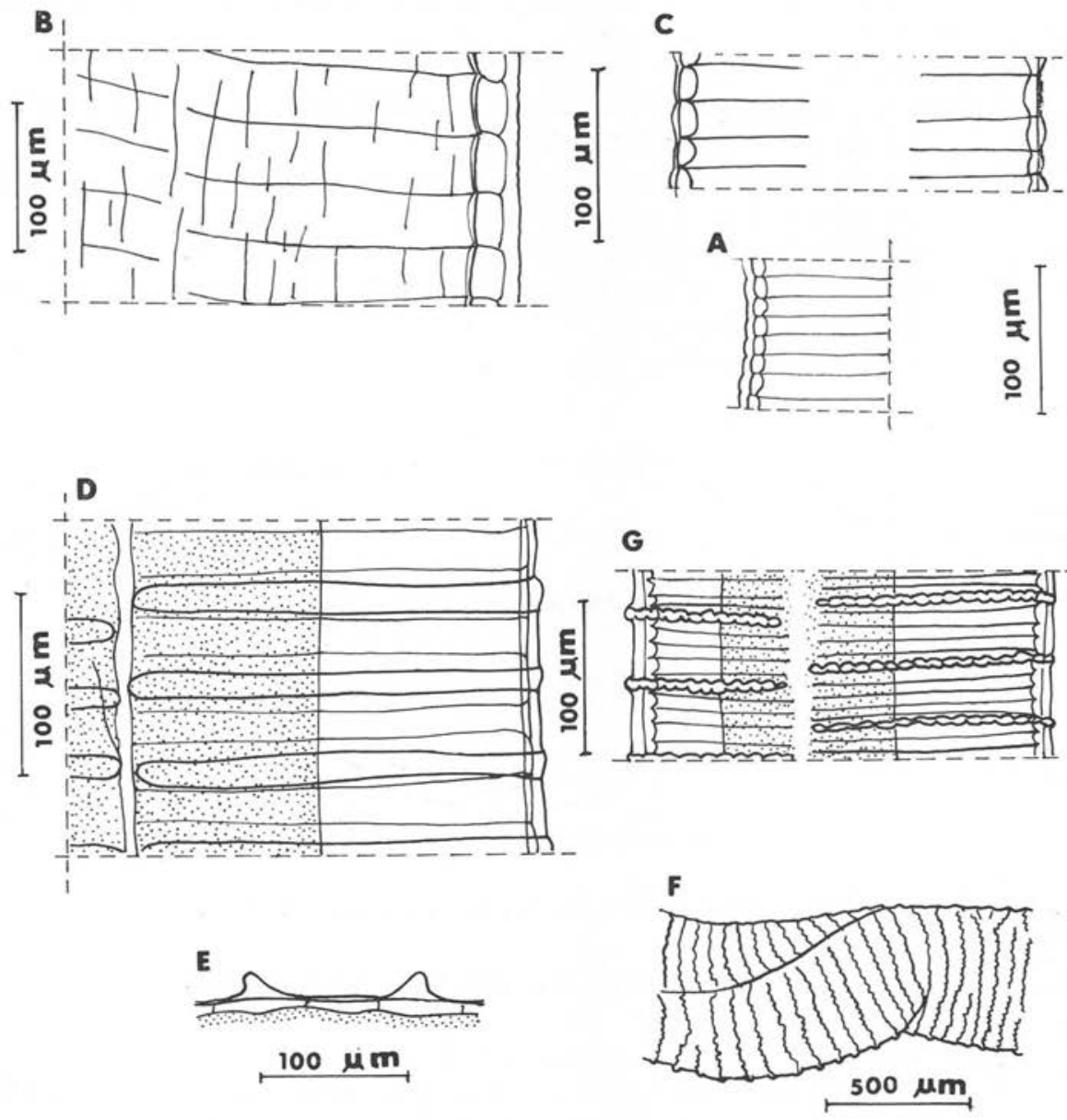

FIG. 14. - Cuticule , vue latérale; $\mathrm{A}: O$. ochengi ; B : O. schulzkeyi ; C : O. dukei ; D : O. volvulus; $\mathrm{E}$ et $\mathrm{F}: O$. gibsoni; $\mathrm{G}: 0$. sweetae. 
36 - (37) Microfilaire épaisse de 6-7 $\mu \mathrm{m}$ à tête trapézoïdale ou arrondie-oblique suivant l'orientation ( $\mathrm{fig} .15 \mathrm{~A}$ ).

Onchocerque de petite taille $(20 \mathrm{~cm}$ pour la ㅇ).

Cuticule o mince (5 à $12 \mu \mathrm{m})$.

Spicule gauche long de 190-220 $\mu \mathrm{m}$.

Spicule droit long de 70-90 $\mu \mathrm{m}$, à talon très saillant formant un angle aigu, et parfois orné d'aspérités cuticulaires ( $\mathrm{fig} .17 \mathrm{~A}$ ).

Parasite intradermique ventral des Bovins, Bos taurus et B. indicus en Afrique.

O. ochengi Bwangamoi, 1969.

37 - (36) Microfilaire d'épaisseur inférieure ou égale à $5 \mu \mathrm{m}$.

38 - (39) Extrémité antérieure de la + mince et non enroulée sur une distance de $5 \mathrm{~cm}$ (fig. 13B).

우 longue de $100 \mathrm{~cm}$.

Cuticule 우 épaisse $(25-30 \mu \mathrm{m})$, à rides longitudinales externes (fig. 14B).

Spicule gauche long de $180-200 \mu \mathrm{m}$.

Spicule droit long de $76-80 \mu \mathrm{m}$, au talon formant un angle droit et à la spatule très courte (fig. 17B).

Microfilaire longue de 225-265 $\mu \mathrm{m}$ sur 3,5-4 $\mu \mathrm{m}$ de large.

Parasite intradermique ventral d'un Bovidé, Hippotragus equinus, en savane africaine (Togo).

O. schulzkeyi Denké et Bain, 1981.

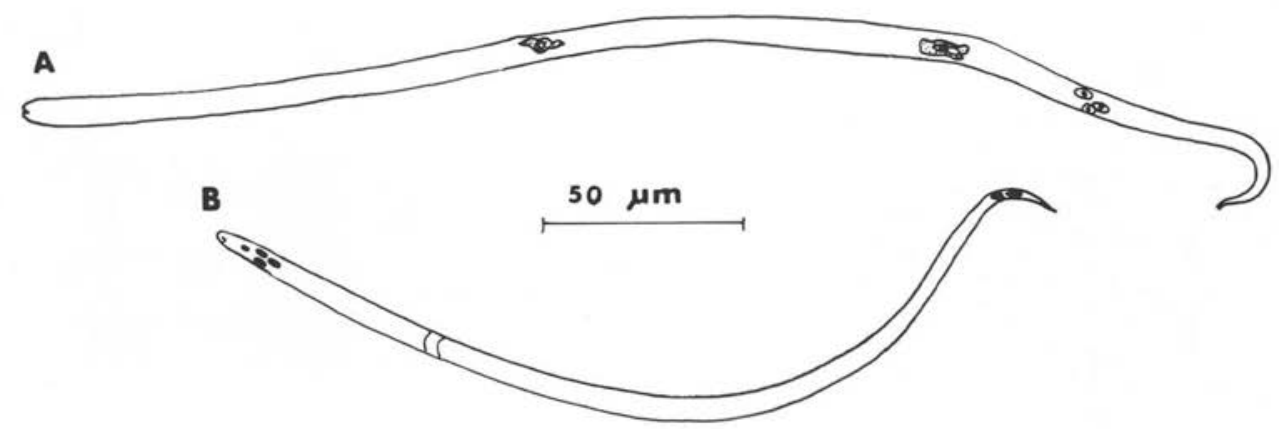

FIG. I5. - Microfilaire; A : O. ochengi; B : O. dukei.

39 - (38) Extrémité antérieure de la 우 trapue et enroulée ( fig. 13C).

우 longue de $35 \mathrm{~cm}$.

Cuticule moins épaisse (12-22 $\mu \mathrm{m})$, sans rides longitudinales.

$\widehat{o}$ inconnu.

Microfilaire longue de $250 \mu \mathrm{m}$ et large de $4,8 \mu \mathrm{m}$.

Parasite sous-cutané dans la région sacrale d'un Bovidé, Kobus (Adenota) kob, en savane africaine (Togo).

O. hamoni Denké et Bain, 1981. 
40 - (35) Cuticule + à côtes.

41 - (44) Côtes peu élevées et non sinueuses.

Deux stries par intercôtes ( $f i g .14 C, D$ ).

42 - (43) Côtes aussi larges que les intercôtes (fig. 14C).

Microfilaire courte, effilée dans le tiers antérieur (fig. 15B).

Région postérieure o épaisse $(400 \mu \mathrm{m})$ et enroulée (fig. 16 A).

Spicule gauche long de 180-210 $\mu \mathrm{m}$.

Spicule droit long de 73-90 $\mu \mathrm{m}$, à talon peu saillant.

Microfilaire longue de 225-265 $\mu \mathrm{m}$ sur $5 \mu \mathrm{m}$ de large.

Parasite du tissu conjonctif sous-cutané et péri-musculaire ventral de Bovins, B. taurus et B. indicus, en Afrique.

O. dukei Bain, Bussiéras, Amégée, 1974.
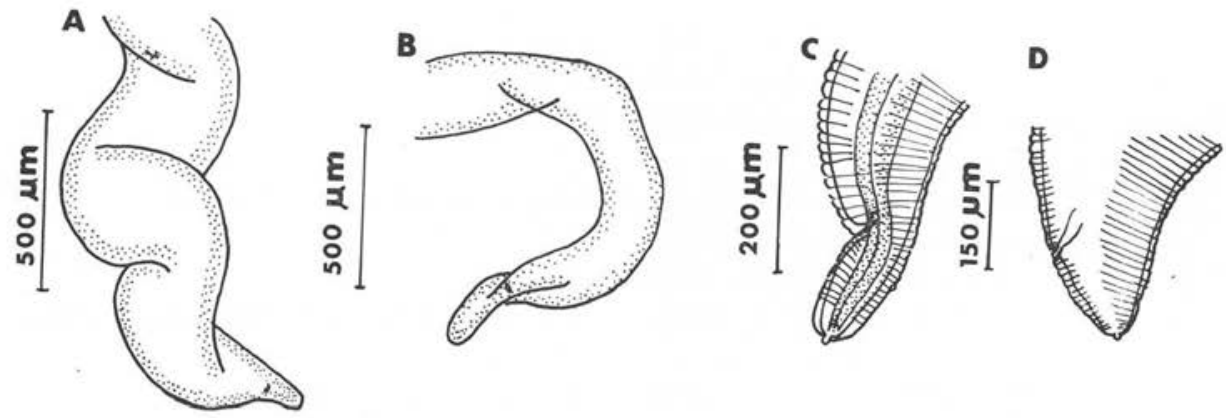

Fig. I6. - Région caudale $\$$; A : O. dukei; B: O. volvulus; C : O. gibsoni; D : O. sweetae.

43 - (42) Intercôtes plus larges que les côtes (fig. 14D).

Microfilaire longue, au tiers antérieur aussi large que le reste du corps.

Région postérieure + moins large $(200 \mu \mathrm{m})$ et peu enroulée ( $f i g .16 \mathrm{~B})$.

Spicule gauche long de $210-310 \mu \mathrm{m}$.

Spicule droit long de $75-140 \mu \mathrm{m}$, à spatule longue.

Microfilaire longue de 315-360 $\mu \mathrm{m}$ sur 6-7 $\mu \mathrm{m}$ de large.

Parasite du conjonctif sous-cutané de l'homme, en Afrique, au Yemen, en Amérique Centrale et du Sud.

O. volvulus (Leuchart, 1893).

44 - (41) Côtes saillantes et sinueuses (fig. $14 \mathrm{~F}$ et $\mathrm{G}$ ).

45 - (46) Deux stries par intercôte (fig. 14E).

$\widehat{o}<5 \mathrm{~cm}$ de long.

Microfilaire supérieure à $250 \mu \mathrm{m}$ de long et inférieure à $4 \mu \mathrm{m}$ de large

Queue de la ㅇ cylindrique.

Spicule gauche long de 180-195 $\mu \mathrm{m}$.

Spicule droit long de 72-90 $\mu \mathrm{m}$ au talon bien marqué ( fig. 17C).

Microfilaire longue de 260-295 $\mu \mathrm{m}$ sur 3,6-3,7 $\mu \mathrm{m}$ de large.

Parasitesous-cutané et périmusculaire ventral de Bovins, B.taurus. 

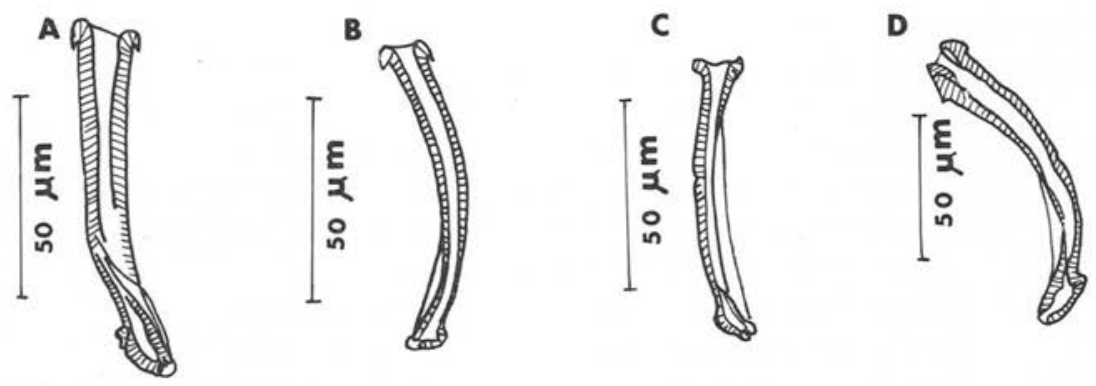

FIG. I7. - Spicule droit; A :O. ochengi; B : O. schulzkeyi; C : O. gibsoni ; D : O. sweetae.

et B. indicus, en Inde, Asie et Australie.

O. gibsoni Cleland et Johnston, 1910.

46 - (45) Quatre stries par intercôtes (fig. 14G).

o $>6 \mathrm{~cm}$ de long.

Microfilaire inférieure à $250 \mu \mathrm{m}$ de long et supérieure à $4 \mu \mathrm{m}$ de large.

Queue de la + conique.

Spicule gauche long de $280-415 \mu \mathrm{m}$.

Spicule droit long de 100-115 $\mu \mathrm{m}$, à talon formant un angle aigu saillant et à spatule longue (fig. 17D).

Microfilaire longue de 215-245 $\mu \mathrm{m}$ sur 4-5 $\mu \mathrm{m}$ de large.

Parasite intradermique de Bovins, Bubalus bubalis en Inde, Asie et Australie.

O. cebei Galliard, 1937 (=O. sweetae Spratt et Moorhouse, 1971). 


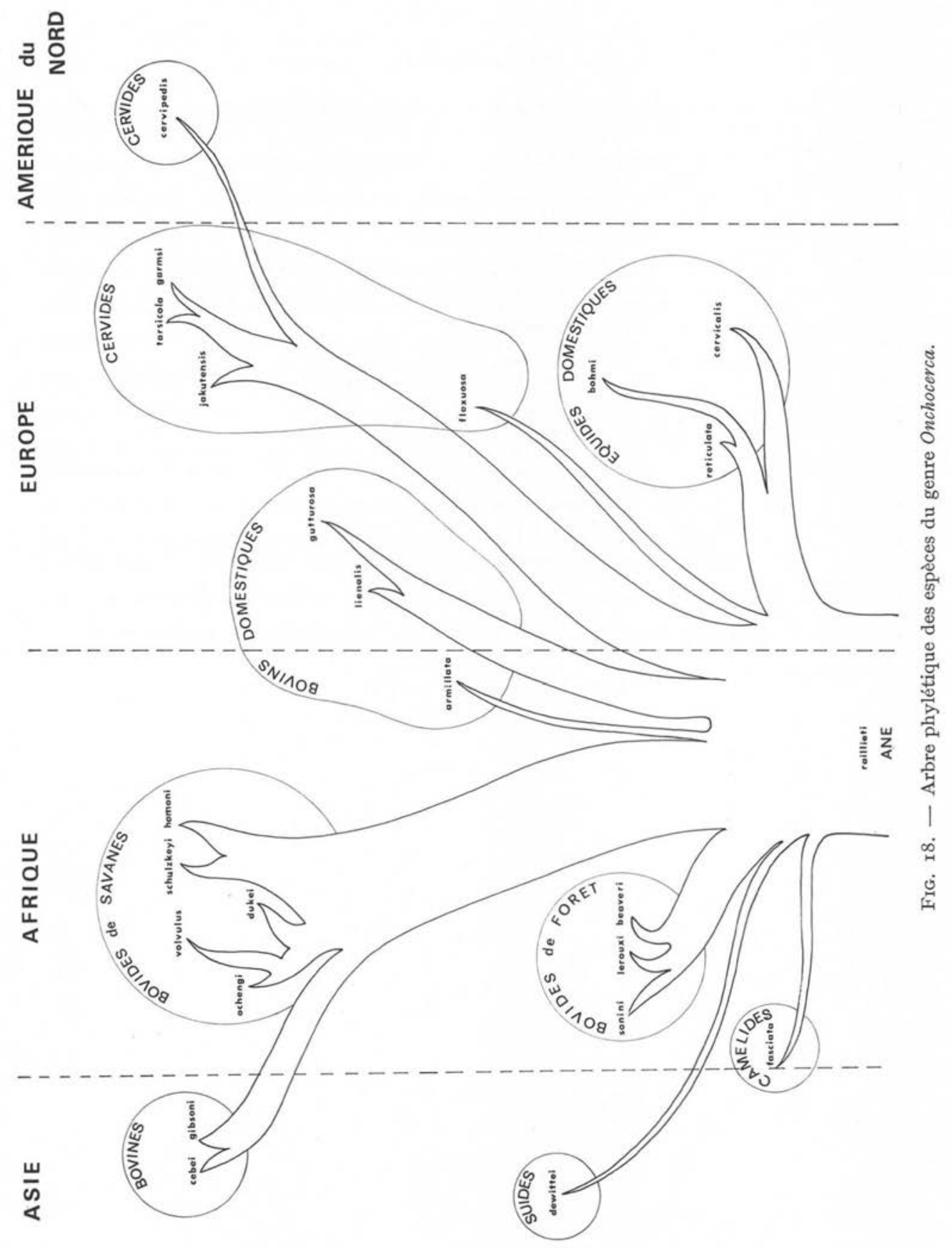




\section{BIBLIOGRAPHIE}

Bain O., Schulz-Key H. : Les onchocerques du Cerf européen : rediscription d'O. flexuosa (Wedl, $\mathrm{r} 856$ ) et description d'O. tubingensis n. sp. et $O$. tarsicola n. sp. Tropenmed. Parasit., 1974, $25,437-449$.

BaIN O., PETIT G. : Redescription du stade infestant d'Onchocerca cervicalis R. et H., I9Io. Ann. Parasitol. Hum. Comp., 1978, 53, 315-318.

Barn O. : Transmission de l'onchocerque bovine, Onchocerca gutturosa, par Culicoides. Ann. Parasitol. Hum. Comp., 1979, $54,483-488$.

Bain O., Beveridge I. : Redescription d'Onchocerca gibsoni C. et J., I9Io. Ann. Parasitol. Hum. Comp., 1979, $54,69-80$.

Bain O., Chabaud A. G., Wanantasamruad P., Nabhitabhata J. : Onchocerca sweetae chez le buffle en Thailande. Ann. Parasitol. Hum. Comp., 1980, S5, 253-259.

BaIN O., NASHER A. K. : Redescription de l'Onchocerque du dromadaire, $O$. fasciata $R$. et H., r9ro. Ann. Parasitol. Hum. Comp., 1981, sous presse.

Chabaud A. G., Petter A. : Remarques sur l'évolution des papilles cloacales chez les Nématodes Phasmidiens parasites de Vertébrés. Parassitologia, 1961, 3, 51-70.

Denké A. M., Barn O., : Deux nouvelles Onchocerques nodulaires chez des Bovidés sauvages, en Haute Volta. Ann. Parasitol. Hum. Comp., I981, 56, 339-347,.

Durette-Desset M. C. : Évolution des Nématodes Héligmosomes en rapport avec celle de leurs hôtes fondamentaux, les Microtidae. C. R. Acad. Sci., Paris, 1967, 265, 1500-1503.

Durette-Desset M. C., Kinsella M. C., Forrester D.' J. : Arguments en faveur de la double origine des Nématodes néarctiques du genre Heligmosomoides Hall, 1916. Ann. Parasitol. Hum. Comp., 1972, 47, 365-382.

Gubanov N. M. : (Helminth fauna of economically important mammals in the Yakut A.S.S.R.). Izdatelsvo Nauka, Moscow, 1964, $164 \mathrm{p}$.

Hibler C. P. : Description of the microfilaria of Wehrdikmansia cervipedis (Wehr et Dikmans, 1935) and observations on its location in Arizona Deer. Bull. Wildlife Dis. Assoc., 1965, I, 44-48.

Railitet A., Henry A. : Les onchocerques, Nématodes parasites du tissu conjonctif. C. R. Soc. Biol., 1910, 58, 248-25I.

SUPPERER R. : Filariosen der Pferde in Osterreich. Wien. Tier. Monatss., 1953, 40, 193-220.

WEHR E. E., Dikmans G. : New Nematodes (Filariidae) from North American ruminants. Zool. Anz., 1935, 110, 202-208. 